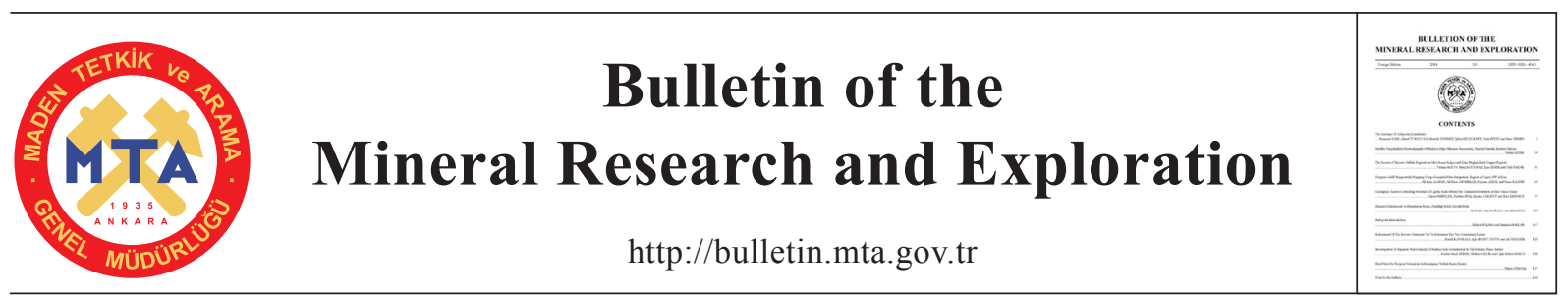

\title{
INVESTIGATION OF IRRIGATION WATER QUALITY OF SURFACE AND GROUNDWATER IN THE KÜTAHYA PLAIN, TURKEY
}

\author{
Berihu Abadi BERHE ${ }^{a^{*}}$, Mehmet ÇELIKK ${ }^{\mathrm{b}}$ and Uğur Erdem DOKUZ ${ }^{\mathrm{a}}$ \\ a Ankara Üniversitesi, Fen Bilimleri Enstitüsü, Jeoloji Mühendisliği Anabilim Dall, 06100, Tandoğan, Ankara \\ ${ }^{b}$ Ankara Üniversitesi, Mühendislik Fakültesi, Jeoloji Mühendisliği ABölümü, 06100, Tandoğan, Ankara
}

Keywords:

Water Chemistry, Water Quality, Hydrochemical Facies, Chemical Index, Agricultural Irrigation, Kütahya Plain

\begin{abstract}
The Kütahya plain is one of the plains in Kütahya/Turkey, where drinking, agricultural and industrial water supplies are highly dependent on groundwater resources. The local population largely depends on water from alluvial shallow aquifer waters and some on the Felent and Porsuk rivers. Appraisal of surface and groundwater quality is extremely important to make sure the sustainable use of it for drinking, agricultural, and industrial purposes. The chemical quality of surface and groundwater of Kütahya plain has been studied in detail in order to have better understanding of potential water quality. A total of 21 groundwater samples and 6 surface water samples were collected in and around the plain. The relative abundance of major ions (meq/l) for most of the water samples were $\mathrm{Ca}^{2+}>\mathrm{Mg}^{2+}>\left(\mathrm{Na}^{+}+\mathrm{K}^{+}\right)$ for cations and $\mathrm{HCO}_{3}^{-}>\mathrm{SO}_{4}^{2-}>\mathrm{Cl}^{-}$for anions. Five hydrochemical facies have been identified based on the major ion chemistry of the surface and groundwater of this area. However, based on hydrochemical facies, the type of water that predominates in the study area is Ca$\mathrm{Mg} / \mathrm{Mg}-\mathrm{Ca}-\mathrm{HCO}_{3}$ type during both December 2013 and June 2014. There is no significant change in the hydrochemical facies noticed during the two sampling periods. The chemical Index such as Sodium Absorption Ratio (SAR), Residual Sodium Carbonate (RSC), Sodium Percentage (\%Na), Permeability Index (PI), Kelley Index (KI), Magnesium Ratio (MR), Potential Salinity (PS) and Total Hardness (TH) were calculated. The results indicated that SAR, RSC and KI values revealed $100 \%, \% \mathrm{Na}$ value revealed $92.6 \%$, PI and PS values revealed $85.2 \%$ and MR value revealed $66.7 \%$ of water samples are within the safe limit suitable for irrigation. To sum up, the quality of surface and groundwater of Kütahya plain in general was suitable for irrigation.
\end{abstract}

\section{Introduction}

Groundwater plays an important role in human life and development. The Safe portable water is absolutely essential for healthy living. About $80 \%$ diseases of the world population and more than one-third of the deaths in the developing countries are due to contamination of water (WHO 1993). Subsurface water is ultimate and most suitable fresh water resource for human consumption in both urban as well as rural areas. Rapid growth of population, development of agriculture and industrial activities especially in alluvial plain aquifers caused an intense increase in water consumption. The chemical constituents of irrigation water can affect plant growth directly through toxicity or deficiency, or indirectly by altering plant availability of nutrients (Ayers and Westcot, 1985; Rowe and Abdel-Magid 1995). Capillary pressure is the main cause for increasing amount of salty waters in vadoz zone and hence the nature of upper part of the soil zone changes into more saline (Çelik et al., 2008).

Irrigation water quality is generally evaluated by some determining factors such as Sodium Absorption Ratio (SAR), Residual Sodium Carbonate (RSC), Sodium Percentage $(\% \mathrm{Na})$, and Electrical Conductivity (EC). Along with the these indicators,

\footnotetext{
* Corresponding author: Berihu Abadi Berhe, berhag2000@gmail.com
} 
some additional indices to categorize the waters for irrigation like Permeability Index (PI), Kelley Index (KI) and Total Hardness (TH) are also important.

The location of this study area was Kütahya district of Kütahya plain. It is located in downstream of Köprüören plain and upstream of Porsuk dam where the people of Eskişehir and Kütahya use as main source of water for both irrigation and domestic purposes. In the study area, people of this district and surrounding villages are the pioneer users of surface and groundwater for drinking and irrigation Purpose.

The interactions between agricultural irrigation, surface water and groundwater resources are always very close. The agricultural activities and industrial establishments in Kütahya city such as: sugar factories, nitrogen factories and leather industries are responsible for disposing treated and untreated effluents in the natural drainage system of Felent and Porsuk rivers. This paper describes the surface and subsurface water chemistry of shallow aquifers affected by agricultural activities and industrial establishments at Kütahya plain, Kütahya, Turkey.
Therefore, the present study was mainly conducted to measure and analyze the irrigation water quality parameters of Felent and Porsuk Rivers and groundwater of Kütahya plain that could potentially impact food safety of irrigation crops.

\subsection{Study Area}

The study area, Kütahya plain including its catchment is bounded by (UTM ) coordinates 225000 268000 E longitude and $4345000-4380000$ N latitude with an area of around $93 \mathrm{~km}^{2}$ and its cathment covers about $530 \mathrm{~km}^{2}$. Location map of study area shows in figure 1. The area is characterized by hills in the southwest and western parts. The highest elevation in the hilly area is $1764 \mathrm{~m}$ above sea level, whereas, much of the plain flat-lying, with height, typically, 920 to 950 $\mathrm{m}$ above sea level. The maximum length between the northwestern to southeastern tips and width of the plain is about $25.0 \mathrm{~km}$ and $5.5 \mathrm{~km}$ respectively. The natural surface drainage within the study area is generally towards the two dominant and perennial rivers: Felent and Porsuk Rivers. Felent River is fed by Enne dam located on the northwest of the Kütahya plain.

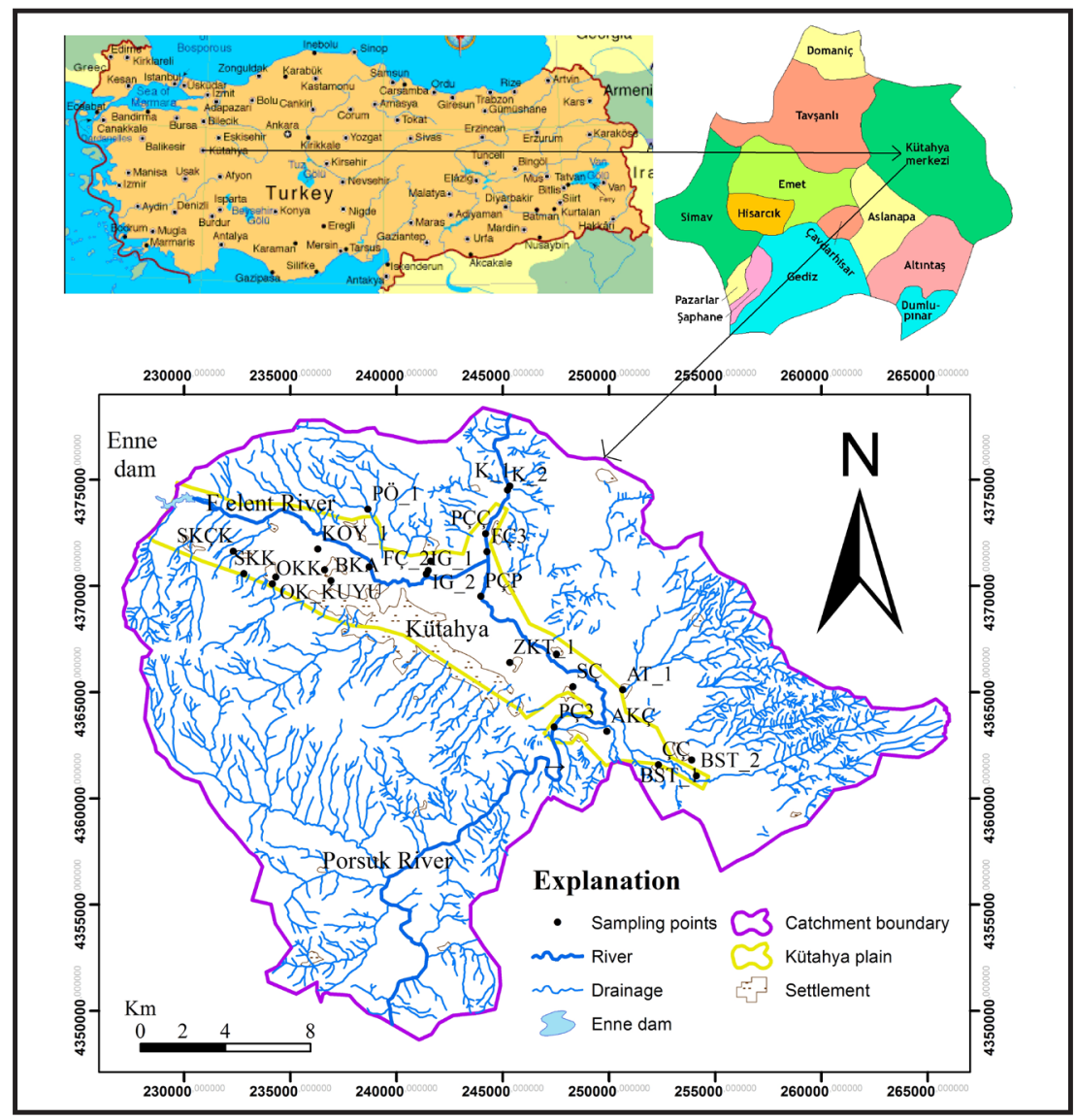

Figure 1- Location map of the study area and the sampling sites. 
Kütahya has a warm summer continental climate, with cold and snowy winters and warm and dry summers. Rainfall occurs mostly during the spring and autumn. According to the data recorded at Kütahya Meteorological Station between the years 1975 and 2011, 75\% of total rainfall occurs during the period of November - May and the annual average precipitation is about $543.85 \mathrm{~mm}$. The average annual air temperature is $10.8^{\circ} \mathrm{C}$ with a maximum in July to August and a minimum in December to January.

\subsection{Geological and Hydrogeological situation}

The basment of of the study area Paleozoic age rocks. On top of these rocks Mesozoic, Neogene and Quatrernary rocks are exposed (Figure 2).

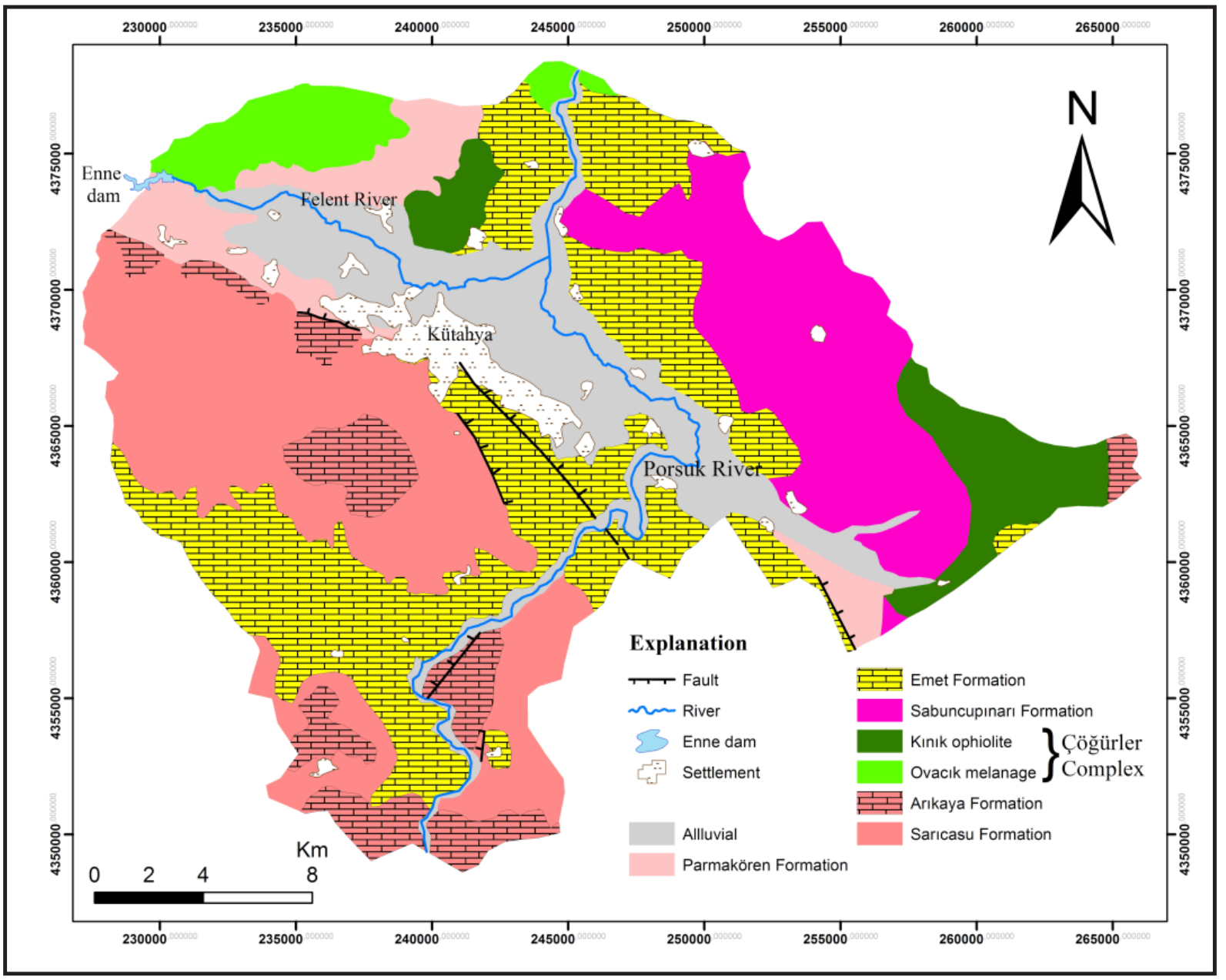

Figure 2- Geological map of the study area (modified from DSİ 1981, 2003; Özburan, 2009).

The oldest lithologic unit cropping out in the south west part of study area is the Sarıcasu formation of Paleozoic age, composed of schists, calc schist, quartz schist and crystallized limestone. At the top of the Saricasu formation is the Arikaya formation of Upper Permian-Lower Triassic age, Marble. Unconformably overlying these Paleozoic schists and marble, are the outcropping Mesozoic age of Çöğürler Comlexes which is composed of low-grade regional metamorphism, and schistserpentinizated periodite and ophiolite-greenschist (DSI 1981 and 2003, Özburan, 2009). Crystalline limestones which we ascribe to the Mesozoic, unconformably overlie the Çayca Tuf and at the top, it is also unconformably covered by Plieistocene age of Parmakören Formation and Alluvials. Quaternary alluvium, consisting of fluviatile pebble, gravel, sand, clay, and silt, is widely distributed in this plain and has a maximum depth of 100 meters.

Quaternary sediments are the most important formations over a greater part of the study area specially for irrigation purpose. The groundwater occurs in the unconsolidated quaternary sediments dominated by gravel and sand. 
In the plain the quaternary aquifer's hydraulic conductivity $(\mathrm{K})$ and transmissivity $(\mathrm{T})$ ranges $0.01-336 \mathrm{~m} /$ day and $1.61-1010 \mathrm{~m}^{2} /$ day and with a mean values of $44.42 \mathrm{~m} /$ day and $213.48 \mathrm{~m}^{2} /$ day, respectivelly (Berhe et al., 2014).

The Parmakören formation is also another water bearing formation exposed in western part of the plain. The sandstone and gravelstone of this formation has hydraulic conductivity that ranges from 0.02 to $0.44 \mathrm{~m} /$ day with a mean value of $0.11 \mathrm{~m} /$ day, and transmissivity varies from 1.56 to $26.92 \mathrm{~m}^{2} /$ day and its mean value is $8.59 \mathrm{~m}^{2} /$ day (Berhe et al. 2014).

In some boreholes the hydraulic characteristics of limestone aquifer of Emet formation within the area is affected by the dominance of marl lithology. Yield values show that some wells gave very small amount of water: for example $0.7 \mathrm{~L} / \mathrm{sec}$. However, the overall yield and values of aquifer constants are generally high as compared to other aquifers of the study area. In this aquifer there is well that can provide about 84.27 $\mathrm{L} / \mathrm{sec}$ and The calculated hydrualic conductivity $(\mathrm{K})$ and transmissivity $(\mathrm{T})$ of these wells of this formation are ranging between $0.003-26.3 \mathrm{~m} /$ day and 1.11 $2662.01 \mathrm{~m}^{2} /$ day with a mean value of $2.8 \mathrm{~m} /$ day and $350.81 \mathrm{~m}^{2} /$ day, respectivelly (Berhe et al., 2014).

The well opened in Çögürler Complex in the ophiolitic material and has a yield of $7 \mathrm{l} / \mathrm{sec}$. According to the results calculated from pumping test of residual drawdown data, the transmissivity and hydrualic conductivity values were $6.64 \mathrm{~m}^{2} /$ day and $0.034 \mathrm{~m} /$ day, respectively (Berhe et al., 2014).

\section{Material and Method}

Felent and Porsuk rivers were used to collect surface water samples and hand pumps, and wells opend by private and state hydrualic works Turkey were used to collect groundwater samples. A total of 6 surface river samples 3 from each were collected downstream and 21 groundwater samples were collected seasonally from Kütahya alluvial plain and sorrounding in December 2013 and June 2014. In-situ measurements of physicochemical parameters were done in the field using Multi 350i multi-parameter.

Water samples were filtered using 0.45 micron disposable capsule filter and collected in $250 \mathrm{ml}$ polyethylene bottles with poly-seal caps for chemical analysis which have been done at Hacettepe University Water Chemistry Laboratory in Ankara, Turkey, using DIONEX LC25 and ICS-1000 High Performance Ion
Chromotgraphy system and Automatic acid titration burette (for $\mathrm{HCO}_{3}^{-}$and $\mathrm{CO}_{3}{ }^{2-}$ ) using the Standard Methods suggested by the American Public Health Association (APHA, 1989).

Aquachem Version 5.1 software was used to generate some of the important figures presented in this study.

In Arc GIS 10 software, geo-database was used to generate the spatial distribution maps of the chemical indices. The present work used the Inverse Distance Weighted (IDW) method for spatial interpolation of the chemical indices. Inverse Distance Weighted (IDW) is an interpolation technique in which interpolated estimates are made based on values at nearby locations weighted only by distance from the interpolation location (Naoum and Tsanis, 2004).

The parametres which were used in determination of irrigation water quality were all calculated using established standard equations (Table 1).

Electrical conductivity, at $25^{\circ} \mathrm{C}$, can be estimated by multiplying of the sum cations or anions (both in meq/l) by 100 where the accuracy of major ions is less than 5\% (Appelo and Postma, 1994). In this study, electrical conductivity was not done in the field and the EC values of water samples collected during December 2013 was determined according this method (Table 2).

\section{Results}

\subsection{Hydrogeochemistry}

The result of hydrochemical analyses of surface and ground water samples are given in tables 2 and 3. The dominant major ions (meq/l) for most of the water samples were $\mathrm{Ca}^{2+}>\mathrm{Mg}^{2+}>\left(\mathrm{Na}^{+}+\mathrm{K}^{+}\right)$for cations and $\mathrm{HCO}_{3}^{-}>\mathrm{SO}_{4}{ }^{2-}>\mathrm{Cl}^{-}$for anions (Table 4, 5 and Figure 4).

The concentration of cations- $\mathrm{Ca}^{2+}, \mathrm{Mg}^{2+}, \mathrm{Na}^{+}$, $\mathrm{K}^{+}$ions ranged from 21.93 to $165.4 ; 6.89$ to 237.91 ; 0.05 to $105.54 ; 0.52$ to $105.4 \mathrm{mg} / 1$ and anions $\left(\mathrm{HCO}_{3}\right.$ , $\left.\mathrm{SO}_{4}{ }^{2-}, \mathrm{Cl}^{-}\right)$varied from 275.39 to $731.41,0.11$ to 649.43; and 1.63 to $173.94 \mathrm{mg} / \mathrm{l}$, respectively. The analytical precision for measurement of ions was determined by calculating the ionic balance error, which falls within the acceptable limits of $\pm 5 \%$. The temperature of the groundwater ranged from $8.5^{\circ} \mathrm{C}$ to $19.5^{\circ} \mathrm{C}$ with an average value of $14.28^{\circ} \mathrm{C}$ (Tables 2 and 3$)$. 
Table 1- Standard equations used to calculate different irrigation water quality indices (All concentrations are in meq/1, TH is in $\mathrm{mg} / \mathrm{l})$.

\begin{tabular}{|l|l|l|}
\hline \multicolumn{1}{|c|}{ Parameter } & \multicolumn{1}{|c|}{ Formula } & \multicolumn{1}{c|}{ Source } \\
\hline Sodium Adsoption Ratio & $\mathrm{SAR}=\frac{N a^{+}}{\sqrt{\frac{C a^{2+}+M g^{2+}}{2}}}$ & Hem (1991) \\
\hline Permeability Index & $\mathrm{PI}=\left(\frac{N a^{+}+H C 3^{-}}{C a^{2+}+M g^{2+}+N a^{+}}\right) \times 100$ & Doneen (1964) \\
\hline Residaul Sodium Carbonate & $\mathrm{RSC}=\left(\mathrm{CO}_{3}^{-}+\mathrm{HCO}_{3}^{-}\right)-\left(\mathrm{Ca}^{2+}+\mathrm{Mg}^{+2}\right)$ & Ragunath (1987) \\
\hline Magnesium Ratio & $\mathrm{MR}=\frac{M g^{2+} X 100}{C a^{2+}+M g^{2+}}$ & Paliwal (1972) \\
\hline Sodium Percentage & $\mathrm{Na} \%=\left(\frac{N a^{+}+K^{+}}{C a^{2+}+M g^{2+}+K^{+}+N a^{+}}\right) \times 100$ & Tank and Chandel (2010) \\
\hline Kelley Index & $\mathrm{KI}=\frac{N a^{+}}{C a^{2+}+M g^{2+}}$ & Kelly (1963) \\
\hline Potential Salinity & $\mathrm{PS}=\mathrm{Cl}^{-}+0.5 \mathrm{SO}_{4}^{2-}$ & Doneen (1964) \\
\hline Total Hardness & $\mathrm{TH}=\left(2.497 \mathrm{Ca}^{2+}\right)+\left(4.11 \mathrm{Mg}^{2+}\right)$ & Todd (1980) \\
\hline
\end{tabular}

Table 2- Results of chemical analyses of the surface and groundwater samples of the study area (Date of sampling: December 2013 ); Explanation: EC $(\mu \mathrm{S} / \mathrm{cm})$, concentrations $(\mathrm{mg} / \mathrm{l})$, temperature $\left({ }^{\circ} \mathrm{C}\right),{ }^{*}$ surface water, ${ }^{\boldsymbol{D}}$ spring water, $\boldsymbol{\Delta}$ shallow groundwater, $\boldsymbol{\nabla}$ deep groundwater.

\begin{tabular}{|c|c|c|c|c|c|c|c|c|c|c|c|}
\hline Sample No & pH & $\mathbf{T}$ & EC & $\mathbf{K}^{+}$ & $\mathbf{N a}^{+}$ & $\mathrm{Mg}^{2+}$ & $\mathrm{Ca}^{2+}$ & $\mathrm{Cl}^{-}$ & $\mathrm{SO}_{4}^{2-}$ & $\mathrm{HCO}_{3}^{-}$ & \%Error \\
\hline PÇ-3* & 8.23 & 13.8 & 613.0 & 2.61 & 9.74 & 21.32 & 83.25 & 2.36 & 9.05 & 337.57 & 4.75 \\
\hline PÇP* & 8.53 & 13.3 & 665.0 & 3.01 & 10.71 & 22.72 & 85.36 & 6.93 & 24.00 & 355.34 & 0.32 \\
\hline PÇÇ* & 7.70 & 14.9 & 844.5 & 6.29 & 31.97 & 24.92 & 81.02 & 22.16 & 31.79 & 438.25 & -0.82 \\
\hline FÇ-1* & 9.67 & 11.7 & 1104.5 & 6.88 & 23.81 & 59.38 & 106.53 & 28.63 & 79.17 & 479.71 & 3.31 \\
\hline FÇ-2* & 8.72 & 10.3 & 1105.5 & 5.85 & 24.49 & 63.95 & 102.71 & 31.40 & 90.03 & 461.94 & 4.94 \\
\hline FÇ-3* & 7.72 & 15.2 & 1111.0 & 30.51 & 31.55 & 39.79 & 119.66 & 26.42 & 22.34 & 574.47 & 4.15 \\
\hline SKK" & 8.83 & 10.6 & 483.0 & 0.86 & 2.27 & 22.15 & 59.32 & 1.78 & 6.50 & 275.39 & 1.89 \\
\hline OKK" & 8.12 & 13.7 & 539.5 & 1.21 & 3.37 & 52.94 & 21.93 & 3.66 & 16.77 & 284.86 & 4.71 \\
\hline $\mathrm{SÇ} \boldsymbol{\Delta}$ & 7.49 & 17.1 & 664.5 & 2.89 & 9.06 & 24.12 & 86.99 & 5.20 & 18.03 & 355.34 & 2.31 \\
\hline $\mathrm{AKÇ} \boldsymbol{\Delta}$ & 8.15 & 14.4 & 813.0 & 4.71 & 17.16 & 33.96 & 95.26 & 15.43 & 34.63 & 396.8 & 3.78 \\
\hline ÇÇ $\boldsymbol{\Delta}$ & 8.18 & 13.4 & 654.0 & 4.59 & 7.37 & 37.75 & 65.00 & 6.20 & 13.43 & 343.49 & 4.10 \\
\hline BST-1 $\boldsymbol{\Delta}$ & 7.89 & 13.2 & 719.5 & 3.63 & 4.43 & 38.22 & 82.52 & 8.14 & 12.79 & 384.95 & 4.98 \\
\hline BST-2 $\boldsymbol{\Delta}$ & 7.71 & 12.8 & 858.5 & 2.49 & 5.45 & 62.23 & 71.37 & 4.63 & 16.12 & 456.02 & 4.59 \\
\hline AT-1 $\boldsymbol{\Delta}$ & 7.99 & 13.8 & 2071.5 & 158.99 & 68.35 & 105.53 & 118.56 & 94.36 & 153.18 & 621.84 & 4.51 \\
\hline İYKT-1 $\boldsymbol{\Delta}$ & 9.11 & 14.0 & 1985.0 & 129.99 & 69.88 & 82.66 & 152.28 & 92.05 & 187.18 & 586.31 & 4.68 \\
\hline ZKT-1 $\Delta$ & 8.00 & 13.3 & 868.0 & 0.86 & 20.33 & 6.89 & 146.16 & 13.88 & 52.54 & 417.52 & 1.01 \\
\hline KOY-1 $\Delta$ & 7.73 & 8.5 & 864.5 & 2.61 & 10.91 & 45.26 & 92.35 & 15.62 & 27.53 & 417.52 & 3.14 \\
\hline $\mathrm{BK}-\mathrm{A} \boldsymbol{\Delta}$ & 8.34 & 13.0 & 720.0 & 1.25 & 5.53 & 41.38 & 76.77 & 11.35 & 13.84 & 355.34 & 4.58 \\
\hline İG-1 $\Delta$ & 7.87 & 14.2 & 1352.5 & 4.38 & 43.86 & 76.79 & 114.77 & 60.99 & 184.30 & 441.21 & 4.53 \\
\hline İG-2 $\boldsymbol{\Delta}$ & 8.57 & 13.5 & 891.0 & 2.57 & 19.71 & 55.3 & 75.61 & 16.94 & 54.38 & 420.49 & 4.24 \\
\hline
\end{tabular}


Table 2- (continued)

\begin{tabular}{|c|r|r|r|r|r|r|r|r|r|r|r|}
\hline İG-3 & 8.93 & 14.5 & 3135.0 & 8.02 & 105.54 & 237.91 & 154.30 & 173.94 & 649.43 & 731.41 & 2.83 \\
\hline KOYM & 7.74 & 12.2 & 1139.0 & 14.09 & 16.08 & 51.91 & 123.41 & 35.50 & 51.42 & 538.93 & 1.58 \\
\hline K-1 $\boldsymbol{\nabla}$ & 7.92 & 14.5 & 704.5 & 6.53 & 19.94 & 22.95 & 86.74 & 25.48 & 41.09 & 296.12 & 3.34 \\
\hline K-2 $\boldsymbol{\nabla}$ & 8.00 & 14.1 & 793.5 & 7.45 & 19.78 & 40.49 & 76.82 & 20.86 & 60.19 & 331.65 & 4.25 \\
\hline PÖ-1 $\boldsymbol{\nabla}$ & 8.46 & 13.8 & 576.5 & 1.85 & 16.4 & 29.78 & 50.16 & 6.33 & 9.39 & 313.88 & -0.05 \\
\hline SKÇK $\mathbf{\nabla}$ & 8.39 & 10.1 & 795.0 & 1.19 & 5.78 & 16.36 & 133.31 & 6.51 & 9.72 & 432.33 & 4.52 \\
\hline OKKuyu $\mathbf{\nabla}$ & 8.45 & 12.7 & 517.0 & 0.60 & 3.12 & 24.67 & 60.26 & 3.19 & 2.73 & 302.04 & 0.44 \\
\hline
\end{tabular}

Table 3- Results of chemical analyses of the surface and groundwater samples of the study area (Date of sampling: June 2014); Explanation: EC ( $\mu \mathrm{S} / \mathrm{cm})$, concentrations $(\mathrm{mg} / \mathrm{l})$, temperature $\left({ }^{\circ} \mathrm{C}\right),{ }^{*}$ surface water, " spring water, $\boldsymbol{\Delta}$ shallow groundwater, $\boldsymbol{\nabla}$ deep groundwater.

\begin{tabular}{|c|c|c|c|c|c|c|c|c|c|c|c|}
\hline Sample No & pH & $\mathbf{T}$ & EC & $\mathbf{K}^{+}$ & $\mathbf{N a}^{+}$ & $\mathbf{M g}^{2+}$ & $\mathrm{Ca}^{2+}$ & $\mathrm{Cl}^{-}$ & $\mathrm{SO}_{4}^{2-}$ & $\mathrm{HCO}_{3}^{-}$ & \%Error \\
\hline PÇ-3* & 7.58 & 16.9 & 548 & 2.30 & 8.55 & 25.03 & 82.40 & 4.98 & 20.16 & 331.65 & 4.29 \\
\hline PÇP* & 7.54 & 18.5 & 608 & 3.82 & 15.64 & 27.33 & 85.61 & 11.58 & 24.20 & 361.26 & 3.70 \\
\hline PÇÇ* & 7.24 & 18.4 & 762 & 6.53 & 23.87 & 32.04 & 82.95 & 21.68 & 38.14 & 393.83 & 4.78 \\
\hline FÇ-1* & 7.42 & 15.5 & 690 & 5.70 & 17.06 & 33.35 & 86.03 & 14.60 & 52.29 & 373.11 & 4.59 \\
\hline FÇ-2* & 7.52 & 15.4 & 556 & 4.16 & 12.12 & 27.75 & 72.38 & 11.48 & 40.29 & 302.04 & 3.43 \\
\hline FÇ-3* & 7.34 & 19.2 & 672 & 7.03 & 19.74 & 30.96 & 78.65 & 18.71 & 54.64 & 343.49 & 4.76 \\
\hline SKK" & 7.62 & 12.8 & 431 & 0.52 & 1.24 & 26.71 & 59.24 & 1.63 & 6.06 & 284.27 & 3.60 \\
\hline OKK• & 7.35 & 13.9 & 694 & 0.85 & 2.82 & 57.99 & 81.00 & 3.10 & 16.56 & 479.71 & 3.82 \\
\hline $\mathrm{SÇ} \boldsymbol{\Lambda}$ & 7.20 & 17.1 & 559 & 2.19 & 7.54 & 21.41 & 93.36 & 5.11 & 16.95 & 343.49 & 4.38 \\
\hline $\mathrm{AKÇ} \boldsymbol{\Delta}$ & 7.32 & 15.9 & 672 & 3.44 & 14.25 & 35.31 & 91.09 & 11.61 & 32.62 & 393.83 & 3.69 \\
\hline ÇÇ $\wedge$ & 7.47 & 15.4 & 542 & 3.63 & 6.01 & 43.46 & 60.63 & 5.21 & 12.59 & 367.18 & 3.02 \\
\hline BST-1 $\mathbf{\Delta}$ & 7.55 & 16.4 & 629 & 2.26 & 3.68 & 43.18 & 77.69 & 5.96 & 12.62 & 384.95 & 3.78 \\
\hline BST-2 $\triangle$ & 7.32 & 16.2 & 681 & 2.15 & 5.15 & 57.10 & 75.63 & 3.87 & 12.93 & 456.02 & 4.50 \\
\hline AT-1 $\boldsymbol{\Delta}$ & 7.1 & 17.1 & 2050 & 165.99 & 63.35 & 100.53 & 120.56 & 95.36 & 140.18 & 630.84 & 4.51 \\
\hline IYYKT-1 $\Delta$ & 7.10 & 19.5 & 1677 & 105.40 & 57.44 & 85.59 & 146.58 & 89.27 & 173.05 & 581.10 & 3.78 \\
\hline ZKT-1 $\mathbf{\Delta}$ & 7.04 & 18.6 & 822 & 0.60 & 16.73 & 12.42 & 165.40 & 17.00 & 50.88 & 444.17 & 4.72 \\
\hline KOY-1 $\boldsymbol{\Delta}$ & 7.25 & 12.6 & 756 & 1.87 & 9.00 & 48.95 & 97.81 & 14.79 & 24.54 & 444.17 & 3.67 \\
\hline BK-A $\Lambda$ & 7.20 & 14.6 & 625 & 0.89 & 4.39 & 42.40 & 75.78 & 11.48 & 13.40 & 364.22 & 3.58 \\
\hline İG-1 $\Delta$ & 7.16 & 14.3 & 1226 & 3.29 & 39.84 & 83.02 & 128.86 & 83.41 & 231.92 & 438.25 & 2.12 \\
\hline İG-2 $\triangle$ & 7.21 & 12.3 & 767 & 2.10 & 18.59 & 54.37 & 82.82 & 15.96 & 60.84 & 432.33 & 3.54 \\
\hline İG-3 $\Delta$ & 7.19 & 12.5 & 1822 & 5.04 & 73.71 & 173.39 & 124.55 & 110.99 & 430.32 & 651.45 & 2.20 \\
\hline KOYM $\mathbf{\Delta}$ & 7.01 & 12.0 & 789 & 9.44 & 8.18 & 47.64 & 101.53 & 18.54 & 25.58 & 467.86 & 3.73 \\
\hline $\mathrm{K}-1 \boldsymbol{\nabla}$ & 7.13 & 17.6 & 832 & 6.88 & 29.65 & 34.13 & 102.73 & 76.57 & 50.57 & 319.80 & 3.35 \\
\hline $\mathrm{K}-2 \boldsymbol{\nabla}$ & 7.49 & 19.0 & 580 & 5.64 & 11.00 & 36.61 & 59.71 & 13.59 & 27.25 & 296.12 & 3.95 \\
\hline PÖ-1 $\boldsymbol{\nabla}$ & 7.31 & 18.7 & 516 & 1.44 & 14.74 & 33.37 & 53.68 & 6.26 & 9.06 & 313.88 & 3.03 \\
\hline SKÇK $\boldsymbol{\nabla}$ & 7.11 & 13.5 & 667 & 0.73 & 5.42 & 21.79 & 120.93 & 8.38 & 5.21 & 432.33 & 3.06 \\
\hline OK Kuyu $\mathbf{\nabla}$ & 7.08 & 12.2 & 555 & 0.78 & 4.28 & 30.34 & 71.13 & 6.29 & 10.24 & 337.57 & 1.32 \\
\hline
\end{tabular}


The evolution of hydrochemical parameters of groundwater can be understood by plotting the concentration of major cations and anions in the Piper's and Schoeller's diagrams. The most acceptable method to classify and compare water types based on ionic composition is proposed by Piper (1944) by plotting the chemical data on a trilinear diagram (Figure 3). Schoeller (1967) diagram is semi-logarithmic diagram was developed to represent major ion analyses in meq/l and to demonstrate different hydrochemical water types on the same diagram (Figure 4). This type of graphical representation has the advantage that, unlike the Piper diagram, actual water sample concentrations are displayed and compared.

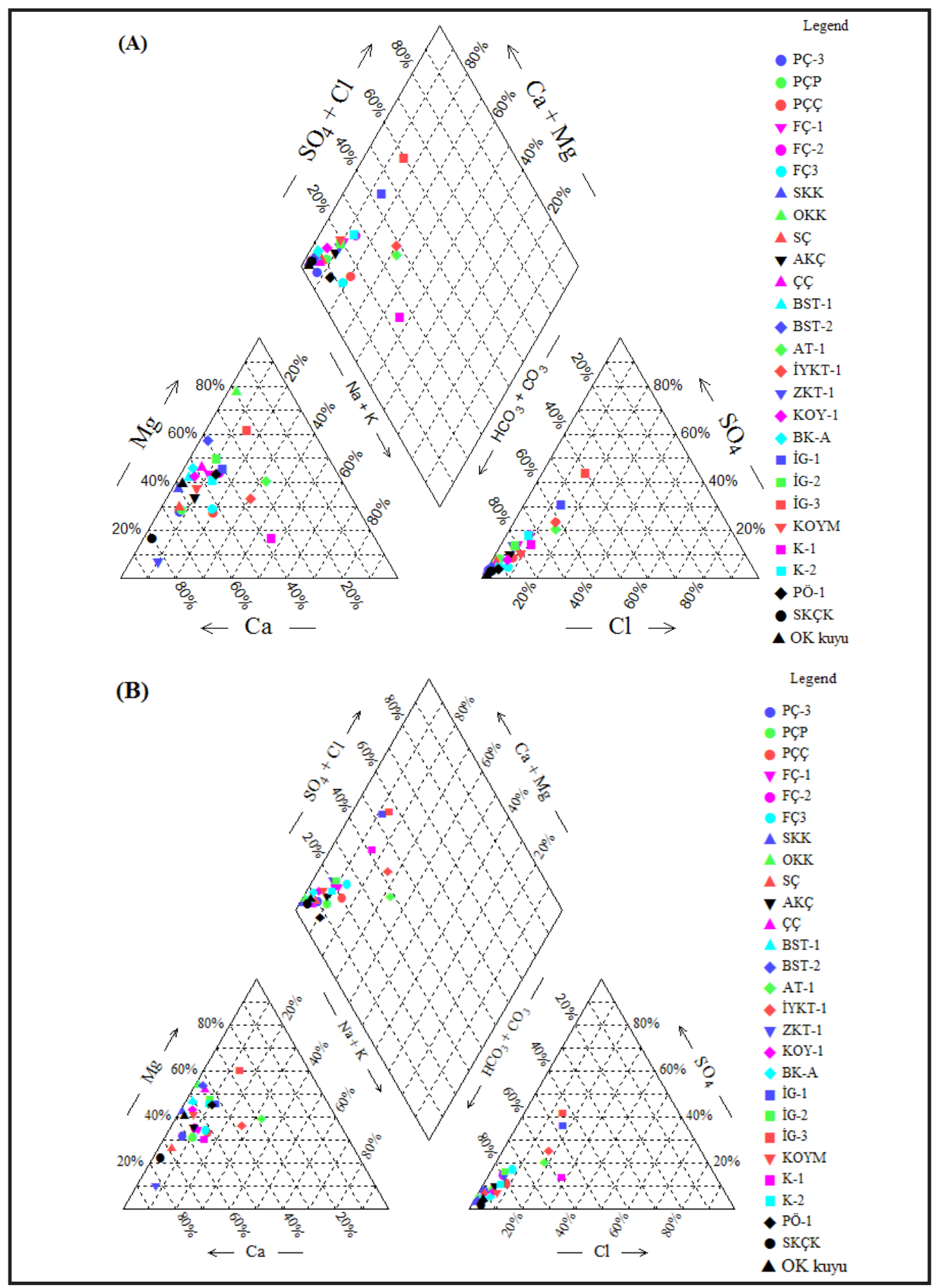

Figure 3- Piper's diagram of surface and groundwater samples (Sampling date- A: December 2013 and B: June 2014). 

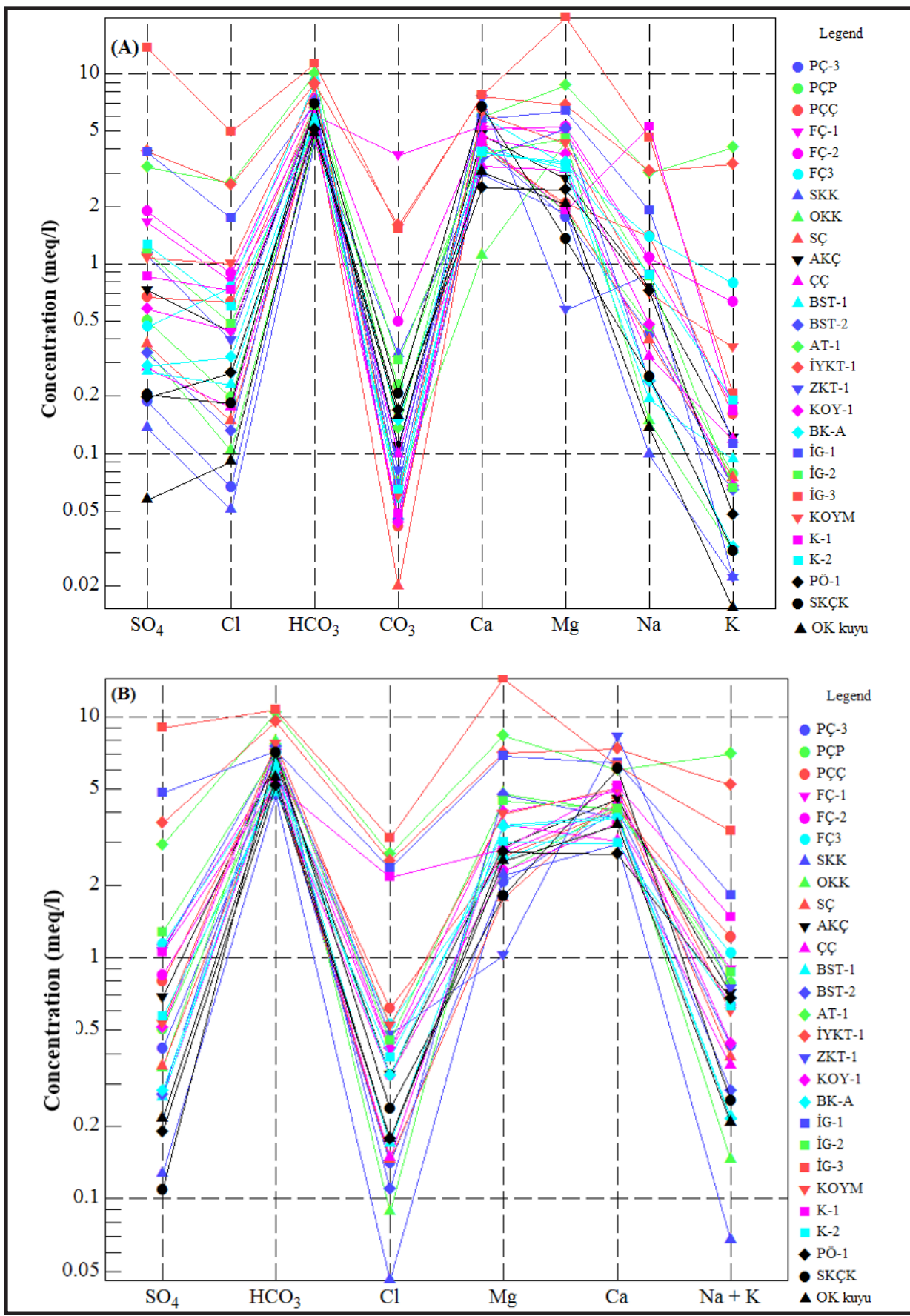

Figure 4- Schoeller diagram of surface and groundwater samples (Sampling date- A: December 2013 and B: June 2014).

The concentration of major ions of surface and groundwater of the study area were plotted in the Piper trilinear diagram to determine the Hydrochemical facies/water type. Hydrochemical facies are, district zones that posses cation and anion concentration categories, a result of rock-water interactions, geology and natural or anthropogenic contamination sources used to describe the type of water that differ in their chemical composition.
Five hydrochemical facies have been identified based on the major ion chemistry of the surface and groundwater of this area. However, based on hydrochemical facies, the type of water that predominates in the study area is $\mathrm{Ca}-\mathrm{Mg} / \mathrm{Mg}-\mathrm{Ca}-$ $\mathrm{HCO}_{3}$ type during both December 2013 and June 2014. Most of the waters of the two seasons are similar types but three water samples (IG-1, IG-3 and K-1) resulted in mixed water types (Tables 4, 5, Figure 3 and 4). 
Table 4- Hydrochemical facies of surface and groundwater samples based on major ions (December 2013 samples).

\begin{tabular}{|c|c|c|c|}
\hline Sample No & Cations & Anions & Hydrochemical facies \\
\hline \multicolumn{4}{|l|}{ Surface waters } \\
\hline PÇ-3, PÇP, PÇÇ, FÇ-1 & $\mathrm{Ca}^{2+}>\mathrm{Mg}^{2+}>\left(\mathrm{Na}^{+}+\mathrm{K}^{+}\right)$ & $\mathrm{HCO}_{3}^{-}>\mathrm{SO}_{4}^{2-}>\mathrm{Cl}^{-}$ & $\mathrm{Ca}-\mathrm{Mg}-\mathrm{HCO}_{3}$ \\
\hline FÇ-2 & $\mathrm{Mg}^{2+}>\mathrm{Ca}^{2+}>\left(\mathrm{Na}^{+}+\mathrm{K}^{+}\right)$ & $\mathrm{HCO}_{3}^{-}>\mathrm{SO}_{4}^{2-}>\mathrm{Cl}^{-}$ & $\mathrm{Mg}-\mathrm{Ca}-\mathrm{HCO}_{3}$ \\
\hline FÇ-3 & $\mathrm{Ca}^{2+}>\mathrm{Mg}^{2+}>\left(\mathrm{Na}^{+}+\mathrm{K}^{+}\right)$ & $\mathrm{HCO}_{3}^{-}>\mathrm{Cl}^{-}>\mathrm{SO}_{4}^{2-}$ & $\mathrm{Ca}-\mathrm{Mg}-\mathrm{HCO}_{3}$ \\
\hline \multicolumn{4}{|l|}{ Spring waters } \\
\hline SKK & $\mathrm{Ca}^{2+}>\mathrm{Mg}^{2+}>\left(\mathrm{Na}^{+}+\mathrm{K}^{+}\right)$ & $\mathrm{HCO}_{3}^{-}>\mathrm{SO}_{4}^{2-}>\mathrm{Cl}^{-}$ & $\mathrm{Ca}-\mathrm{Mg}-\mathrm{HCO}_{3}$ \\
\hline OKK & $\mathrm{Mg}^{2+}>\mathrm{Ca}^{2+}>\left(\mathrm{Na}^{+}+\mathrm{K}^{+}\right)$ & $\mathrm{HCO}_{3}^{-}>\mathrm{SO}_{4}^{2-}>\mathrm{Cl}^{-}$ & $\mathrm{Mg}-\mathrm{Ca}-\mathrm{HCO}_{3}$ \\
\hline \multicolumn{4}{|l|}{ Shallow groundwaters } \\
\hline SÇ, AKÇ, ÇÇ, BST-1, IYKT-1, KOY-1, KOYM & $\mathrm{Ca}^{2+}>\mathrm{Mg}^{2+}>\left(\mathrm{Na}^{+}+\mathrm{K}^{+}\right)$ & $\mathrm{HCO}_{3}^{-}>\mathrm{SO}_{4}^{2-}>\mathrm{Cl}^{-}$ & $\mathrm{Ca}-\mathrm{Mg}-\mathrm{HCO}_{3}$ \\
\hline BST-2, AT-, İG-2 & $\mathrm{Mg}^{2+}>\mathrm{Ca}^{2+}>\left(\mathrm{Na}^{+}+\mathrm{K}^{+}\right)$ & $\mathrm{HCO}_{3}^{-}>\mathrm{SO}_{4}^{2-}>\mathrm{Cl}^{-}$ & $\mathrm{Mg}-\mathrm{Ca}-\mathrm{HCO}_{3}$ \\
\hline İG-1 & $\mathrm{Mg}^{2+}>\mathrm{Ca}^{2+}>\left(\mathrm{Na}^{+}+\mathrm{K}^{+}\right)$ & $\mathrm{HCO}_{3}^{-}>\mathrm{SO}_{4}^{2-}>\mathrm{Cl}^{-}$ & $\mathrm{Mg}-\mathrm{Ca}-\mathrm{HCO}_{3}-\mathrm{SO}_{4}$ \\
\hline ZKT-1 & $\mathrm{Ca}^{2+}>\left(\mathrm{Na}^{+}+\mathrm{K}^{+}\right)>\mathrm{Mg}^{2+}$ & $\mathrm{HCO}_{3}^{-}>\mathrm{SO}_{4}^{2-}>\mathrm{Cl}^{-}$ & $\mathrm{Ca}-\mathrm{HCO}_{3}$ \\
\hline BK-A & $\mathrm{Ca}^{2+}>\mathrm{Mg}^{2+}>\left(\mathrm{Na}^{+}+\mathrm{K}^{+}\right)$ & $\mathrm{HCO}_{3}^{-}>\mathrm{Cl}^{-}>\mathrm{SO}_{4}^{2-}$ & $\mathrm{Ca}-\mathrm{Mg}-\mathrm{HCO}_{3}$ \\
\hline İG-3 & $\mathrm{Mg}^{2+}>\mathrm{Ca}^{2+}>\left(\mathrm{Na}^{+}+\mathrm{K}^{+}\right)$ & $\mathrm{SO}_{4}^{2-}>\mathrm{HCO}_{3}^{-}>\mathrm{Cl}^{-}$ & $\mathrm{Mg}-\mathrm{Ca}-\mathrm{SO}_{4}-\mathrm{HCO}_{3}$ \\
\hline \multicolumn{4}{|l|}{ Deep groundwaters } \\
\hline K-1, K-2, PÖ-1 & $\mathrm{Ca}^{2+}>\mathrm{Mg}^{2+}>\left(\mathrm{Na}^{+}+\mathrm{K}^{+}\right)$ & $\mathrm{HCO}_{3}^{-}>\mathrm{SO}_{4}^{2-}>\mathrm{Cl}^{-}$ & $\mathrm{Ca}-\mathrm{Mg}-\mathrm{HCO}_{3}$ \\
\hline SKÇK & $\mathrm{Ca}^{2+}>\mathrm{Mg}^{2+}>\left(\mathrm{Na}^{+}+\mathrm{K}^{+}\right)$ & $\mathrm{HCO}_{3}^{-}>\mathrm{SO}_{4}^{2-}>\mathrm{Cl}^{-}$ & $\mathrm{Ca}-\mathrm{HCO}_{3}$ \\
\hline OK Kuyu & $\mathrm{Ca}^{2+}>\mathrm{Mg}^{2+}>\left(\mathrm{Na}^{+}+\mathrm{K}^{+}\right)$ & $\mathrm{HCO}_{3}^{-}>\mathrm{Cl}^{-}>\mathrm{SO}_{4}^{2-}$ & $\mathrm{Ca}-\mathrm{Mg}-\mathrm{HCO}_{3}$ \\
\hline
\end{tabular}

Table 5- Hydrochemical facies of surface and groundwater samples based on major ions (June 2014 samples).

\begin{tabular}{|c|c|c|c|}
\hline Sample No & Cations & Anions & Hydrochemical facies \\
\hline \multicolumn{4}{|l|}{ Surface waters } \\
\hline $\begin{array}{l}\text { PÇ-3, PÇP, PÇÇ, FÇ-1, } \\
\text { FÇ-2, FÇ-3 }\end{array}$ & $\mathrm{Ca}^{2+}>\mathrm{Mg}^{2+}>\left(\mathrm{Na}^{+}+\mathrm{K}^{+}\right)$ & $\mathrm{HCO}_{3}^{-}>\mathrm{SO}_{4}^{2->} \mathrm{Cl}^{-}$ & $\mathrm{Ca}-\mathrm{Mg}-\mathrm{HCO}_{3}$ \\
\hline \multicolumn{4}{|l|}{ Spring waters } \\
\hline SKK & $\mathrm{Ca}^{2+}>\mathrm{Mg}^{2+}>\left(\mathrm{Na}^{+}+\mathrm{K}^{+}\right)$ & $\mathrm{HCO}_{3}^{-}>\mathrm{SO}_{4}^{2-}>\mathrm{Cl}^{-}$ & $\mathrm{Ca}-\mathrm{Mg}-\mathrm{HCO}_{3}$ \\
\hline OKK & $\mathrm{Mg}^{2+}>\mathrm{Ca}^{2+}>\left(\mathrm{Na}^{+}+\mathrm{K}^{+}\right)$ & $\mathrm{HCO}_{3}^{-}>\mathrm{SO}_{4}^{2-}>\mathrm{Cl}^{-}$ & $\mathrm{Mg}-\mathrm{Ca}-\mathrm{HCO}_{3}$ \\
\hline \multicolumn{4}{|l|}{ Shallow groundwaters } \\
\hline SÇ, AKÇ, BST-1, IYYKT-1, KOY-1, KOYM & $\mathrm{Ca}^{2+}>\mathrm{Mg}^{2+}>\left(\mathrm{Na}^{+}+\mathrm{K}^{+}\right)$ & $\mathrm{HCO}_{3}^{-}>\mathrm{SO}_{4}^{2-}>\mathrm{Cl}^{-}$ & $\mathrm{Ca}-\mathrm{Mg}-\mathrm{HCO}_{3}$ \\
\hline BST-2, AT-1, İG-2, ÇÇ, & $\mathrm{Mg}^{2+}>\mathrm{Ca}^{2+}>\left(\mathrm{Na}^{+}+\mathrm{K}^{+}\right)$ & $\mathrm{HCO}_{3}^{-}>\mathrm{SO}_{4}{ }^{2-}>\mathrm{Cl}^{-}$ & $\mathrm{Mg}-\mathrm{Ca}-\mathrm{HCO}_{3}$ \\
\hline İG-1, İG-3 & $\mathrm{Mg}^{2+}>\mathrm{Ca}^{2+}>\left(\mathrm{Na}^{+}+\mathrm{K}^{+}\right)$ & $\mathrm{HCO}_{3}^{-}>\mathrm{SO}_{4}{ }^{2-}>\mathrm{Cl}^{-}$ & $\mathrm{Mg}-\mathrm{Ca}-\mathrm{HCO}_{3}-\mathrm{SO}_{4}$ \\
\hline ZKT-1 & $\mathrm{Ca}^{2+}>\mathrm{Mg}^{2+}>\left(\mathrm{Na}^{+}+\mathrm{K}^{+}\right)$ & $\mathrm{HCO}_{3}^{-}>\mathrm{SO}_{4}^{2-}>\mathrm{Cl}^{-}$ & $\mathrm{Ca}-\mathrm{HCO}_{3}$ \\
\hline BK-A & $\mathrm{Ca}^{2+}>\mathrm{Mg}^{2+}>\left(\mathrm{Na}^{+}+\mathrm{K}^{+}\right)$ & $\mathrm{HCO}_{3}^{-}>\mathrm{Cl}^{-}>\mathrm{SO}_{4}^{2-}$ & $\mathrm{Ca}-\mathrm{Mg}-\mathrm{HCO}_{3}$ \\
\hline \multicolumn{4}{|l|}{ Deep groundwaters } \\
\hline $\mathrm{K}-1$ & $\mathrm{Ca}^{2+}>\mathrm{Mg}^{2+}>\left(\mathrm{Na}^{+}+\mathrm{K}^{+}\right)$ & $\mathrm{HCO}_{3}^{-}>\mathrm{Cl}^{-}>\mathrm{SO}_{4}^{2-}$ & $\mathrm{Ca}-\mathrm{Mg}-\mathrm{HCO}_{3}-\mathrm{Cl}$ \\
\hline K-2, PÖ-1 & $\mathrm{Mg}^{2+}>\mathrm{Ca}^{2+}>\left(\mathrm{Na}^{+}+\mathrm{K}^{+}\right)$ & $\mathrm{HCO}_{3}^{-}>\mathrm{SO}_{4}^{2-}>\mathrm{Cl}^{-}$ & $\mathrm{Mg}-\mathrm{Ca}-\mathrm{HCO}_{3}$ \\
\hline OK Kuyu, SKÇK & $\mathrm{Ca}^{2+}>\mathrm{Mg}^{2+}>\left(\mathrm{Na}^{+}+\mathrm{K}^{+}\right)$ & $\mathrm{HCO}_{3}^{-}>\mathrm{Cl}^{-}>\mathrm{SO}_{4}{ }^{2-}$ & $\mathrm{Ca}-\mathrm{Mg}-\mathrm{HCO}_{3}$ \\
\hline
\end{tabular}

\subsection{Assessment of Waters for Irrigation Purpose}

Enormous amounts of dissolved ions in irrigation water affect both agricultural soil physically and chemically and plants growth, thus reducing the productivity. Parameters such as Sodium Adsorption Ratio (SAR), Electrical Conductivity (EC), Residual Sodium Carbonate (RSC), Percent of Sodium ( $\% \mathrm{Na})$, Magnesium Ratio (MR), Permeability Index (PI),
Kelley Index (KI) and Potential Salinity (PS) were used to investigate the suitability of Felent and Porsuk rivers and groundwaters for irrigation. The average values of different parameter indices for rating surface and groundwater quality and its sustainability in irrigation for two differnt seasons were calculated (Table 6) and quality of irrigation water in relation to the different parameters is given by table 7 . 


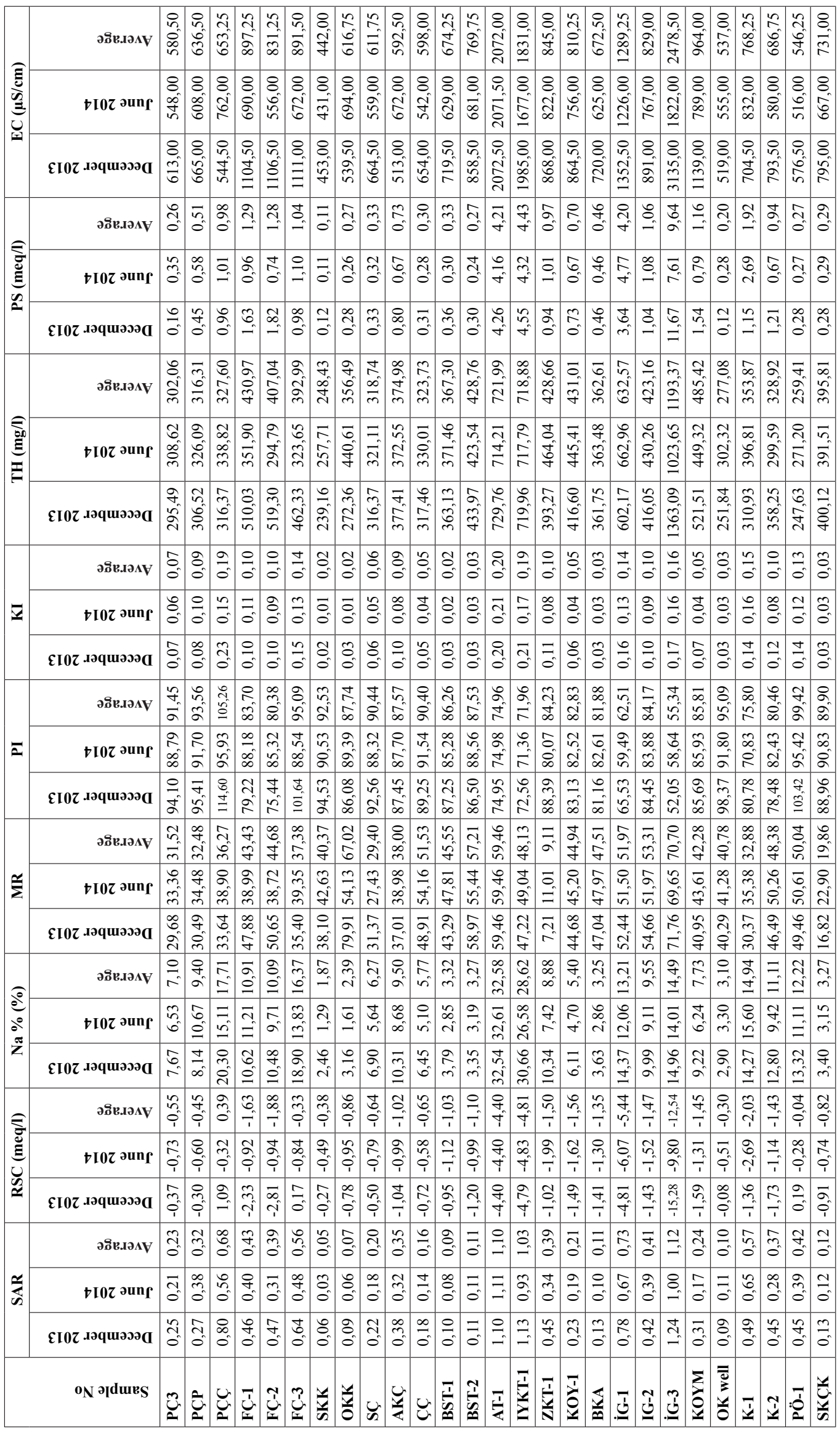


Table 7- Irrigation water quality according to different indices.

\begin{tabular}{|c|c|c|c|}
\hline Parameter & Range & Class & Source \\
\hline \multirow{5}{*}{ EC } & $<250$ & Excellent & \multirow{5}{*}{ Richard (1954) } \\
\hline & $250-750$ & Good & \\
\hline & $750-2000$ & Permissible & \\
\hline & $2000-3000$ & Doubtful & \\
\hline & $>3000$ & Unsuitable & \\
\hline \multirow{5}{*}{$\mathrm{Na} \%$} & $<20$ & Excellent & \multirow{5}{*}{ Todd (1960) } \\
\hline & $20-40$ & Good & \\
\hline & $40-60$ & Permissible & \\
\hline & $60-80$ & Doubtful & \\
\hline & $>80$ & Unsuitable & \\
\hline \multirow{2}{*}{ MR } & $<50$ & Suitable & \multirow{2}{*}{ Paliwal (1972) } \\
\hline & $>50$ & Unsuitable & \\
\hline \multirow{4}{*}{ TH } & $<60$ & Soft & \multirow{4}{*}{ Durfor and Becker (1964) } \\
\hline & $60-120$ & Moderately & \\
\hline & $120-180$ & Hard & \\
\hline & $>180$ & Very hard & \\
\hline \multirow{3}{*}{ RSC } & $<1.25$ & Safe & \multirow{3}{*}{$\begin{array}{c}\text { Aghazadeh and } \\
\text { Mogaddam (2010) }\end{array}$} \\
\hline & $1.25-2.5$ & Marginally suitable & \\
\hline & $>2.5$ & Not suitable & \\
\hline \multirow{5}{*}{ SAR } & $<20$ & Excellent & \multirow{5}{*}{ Todd (1960) } \\
\hline & $20-40$ & Good & \\
\hline & $40-60$ & Permissible & \\
\hline & $60-80$ & Doubtful & \\
\hline & $>80$ & Unsuitable & \\
\hline \multirow{3}{*}{ PI } & $<25$ ( Class III) & Unsuitable & \multirow{3}{*}{ Doneen (1966) } \\
\hline & 25-75 ( Class II) & Good & \\
\hline & $>75($ Class I) & Excellent & \\
\hline \multirow{2}{*}{ KI } & $<1$ & Suitable & \multirow{2}{*}{$\begin{array}{c}\text { Kelley (1940) and Paliwa } \\
\text { (1967) }\end{array}$} \\
\hline & $>1$ & Unsuitable & \\
\hline \multirow[t]{2}{*}{ PS } & $<3$ & Suitable & \multirow{2}{*}{ Doneen (1964) } \\
\hline & $>3$ & Unsuitable & \\
\hline
\end{tabular}

\subsubsection{Sodium Adsorption Ratio (SAR)}

Whenever there is high sodium ion and low in calsium ion concentration in a water used for irrigation purpose, the ion-exchange complex may become saturated with sodium ion which destroys the nature of soil structure, due to the dispersion of the clay particles (Todd, 1980) and affects the plant growth. As indicated in table, the computed SAR values for two different seasons range from 0.03 to 1.24 and all values are within the excellent class
(Table 6 and 7). According to the graph of sodium hazard versus salinty hazard (Wilcox, 1950) all the water sample collected in December 2013 and June 2014 fall into category C2-S1 and C3-S1, indicating low alkali hazards and excellent irrigation water. However, one shallow well water sample collected during december 2013 categorized as C4-S1 type indicating high salinity hazard and low alkali hazard (Figures 5A, B). The average spatial distribution maps of the two seasons is given in figure $6 \mathrm{~A}$ and Northwest of the study area is the safest. 


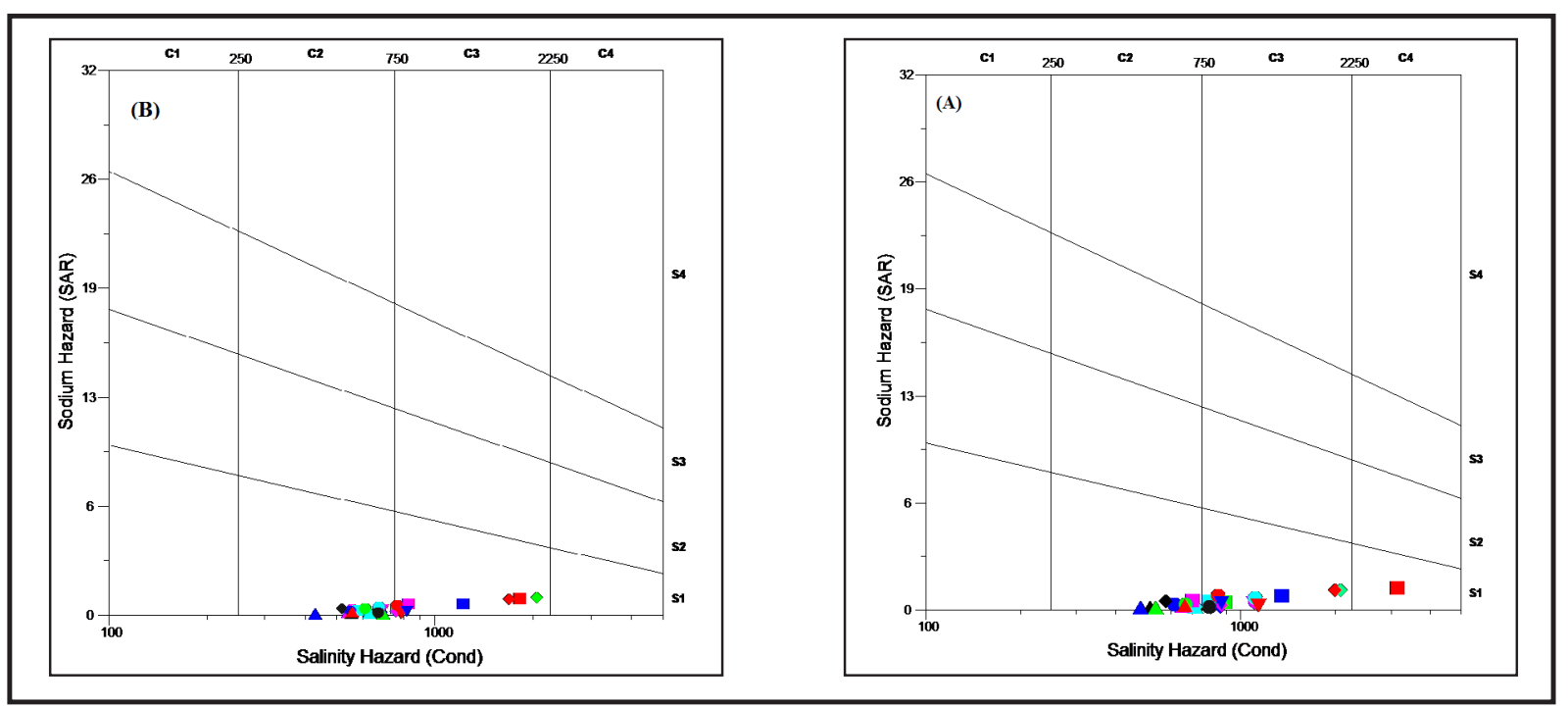

Figure 5- Classification of water samples with respect to salinity and sodium hazard: A) December 2013 and B) June 2014.

\subsubsection{Electrical Conductivity (EC)}

The most important water quality guideline on crop productivity is the salinity hazard as measured by electrical conductivity (Johnson and Zhang, 1990). Figure $6 \mathrm{~B}$ illustrates the spatial distribution of EC over area of study. Majority of the area is characterized by good water quality. The average EC for the two seasons ranges from 442.38 to $2469.69 \mu \mathrm{S} / \mathrm{cm}$ (Figure 6B). According to the the spatial distribution map of EC, the waters in the northwest and southeast of Kutahya plain have a lower salinity hazard.

Based on standard classification systems (Richards, 1954; Todd, 1980), 59\% of the samples falling into the good; $33 \%$ falling into permissible; and the remaining $8 \%$ falling into the doubtful, highly saline signifying high salinity hazard.

\subsubsection{Residual Sodium Carbonate (RSC)}

RSC has been calculated to determine the hazardous effect of carbonate and bicarbonate on the quality of water for agricultural purpose (Aghazadeh and Mogaddam, 2010). Table 6 indicated that the computed RSC values range from -15.28 to 0.39 $\mathrm{meq} / \mathrm{l}$ and water samples are within the safe water category. The spatial distribution of the RSC value are given in Figure 6C and northwestern part of the plain have the lowest RSC values.

\subsubsection{Percent of sodium $(\% \mathrm{Na})$}

Percent of sodium (Wilcox,1955) has been used in determination of groundwater suitability for irrigation, because the concentarion of sodium ion reacts with soil to reduce its permeability (Todd, 1980). The computed $\mathrm{Na} \%$ for the study area ranged from 1.29 to $32.61 \%$. Based on Table 7; and Figure 7D indicate that $92.6 \%$ of the waters from study area are within the excellent class and the rest $7.4 \%$ are within good class. Generally, Northwest of the Kütahya plain is the safest area.

\subsubsection{Magnesium Ratio (MR)}

Magnesium ratio is considered to be one of the most important parametr in determining the suitability of water for irrigation. Excess amount of magnesium in water reduces the growth and yields as the soil becomes more saline (Joshi et al, 2009). The values of MR for all water samples of the study area vary from 7.21 to 79.91 and spatially northwest part of the plain has the lowest values. According to the results $70.4 \%$ of the samples are suitable for irrigational practice (Tables 6, Figure 7E).

\subsubsection{Permeability Index (PI)}

The soil permeability is influenced by long term use of irrigation water and sodium $\left(\mathrm{Na}^{+}\right)$, calcium $\left(\mathrm{Ca}^{2+}\right)$ , magnesium $\left(\mathrm{Mg}^{2+}\right)$, bicarbonate $\left(\mathrm{HCO}_{3}^{-}\right)$content of the soil (Raju, 2007). Doneen (1964) has evolved a formula, permeability index (PI) to measure the soil permeability for assessing the suitability of water for irrigation purposes (Table 1). The PI values range from 52.05 to 114.60 (Table 6) and the results indicate that $85.2 \%$ and $14.8 \%$ of the water samples of the study area fall within class I and class II respectivelly which make the water suitable for irrigation purposes. The 

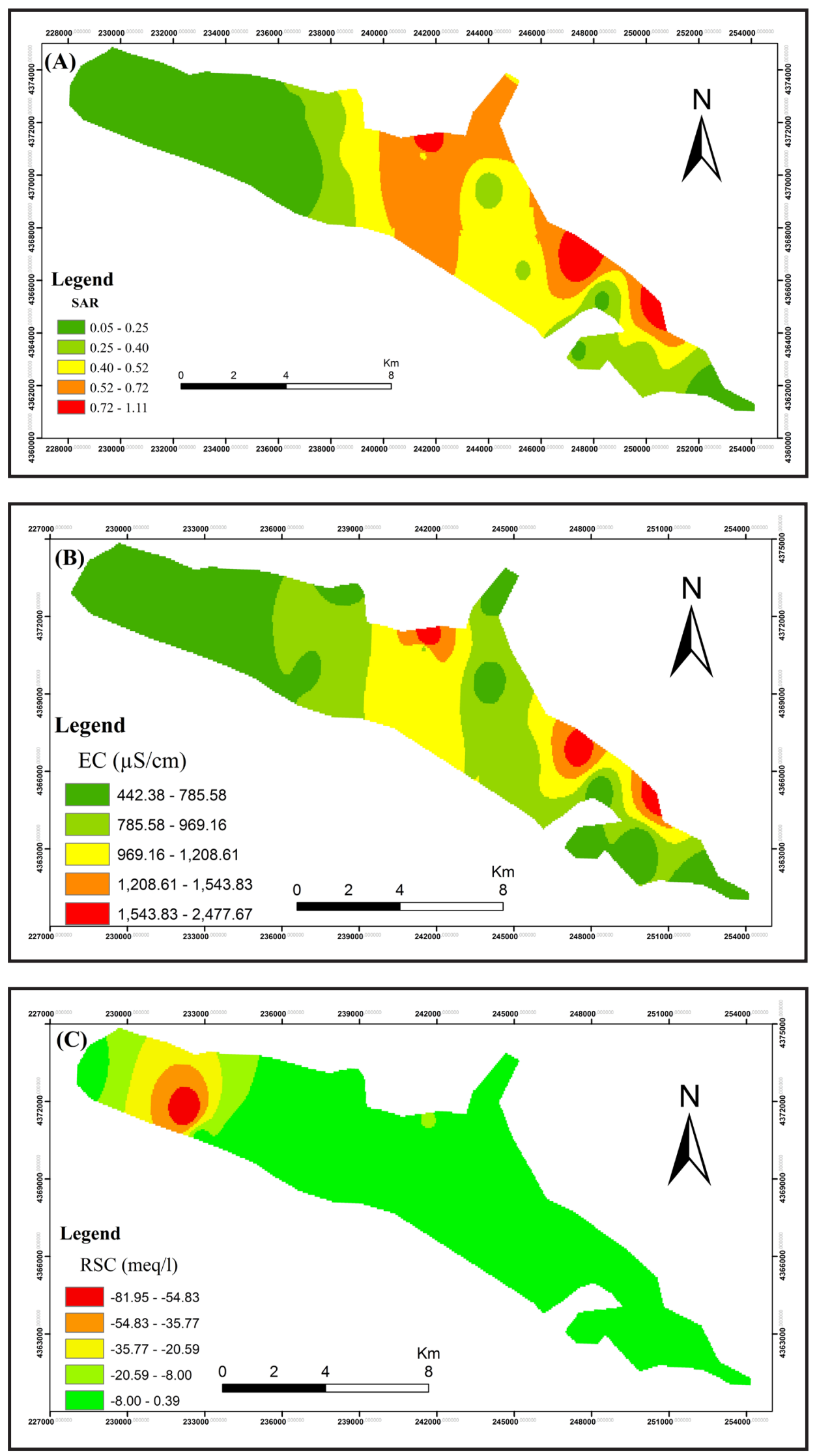

Figure 6- Spatial distribution of average values of two sampling seasons: (A) SAR, (B) EC ( $\mu \mathrm{S}$ / $\mathrm{cm})$ and (C) RSC (meq/l). 

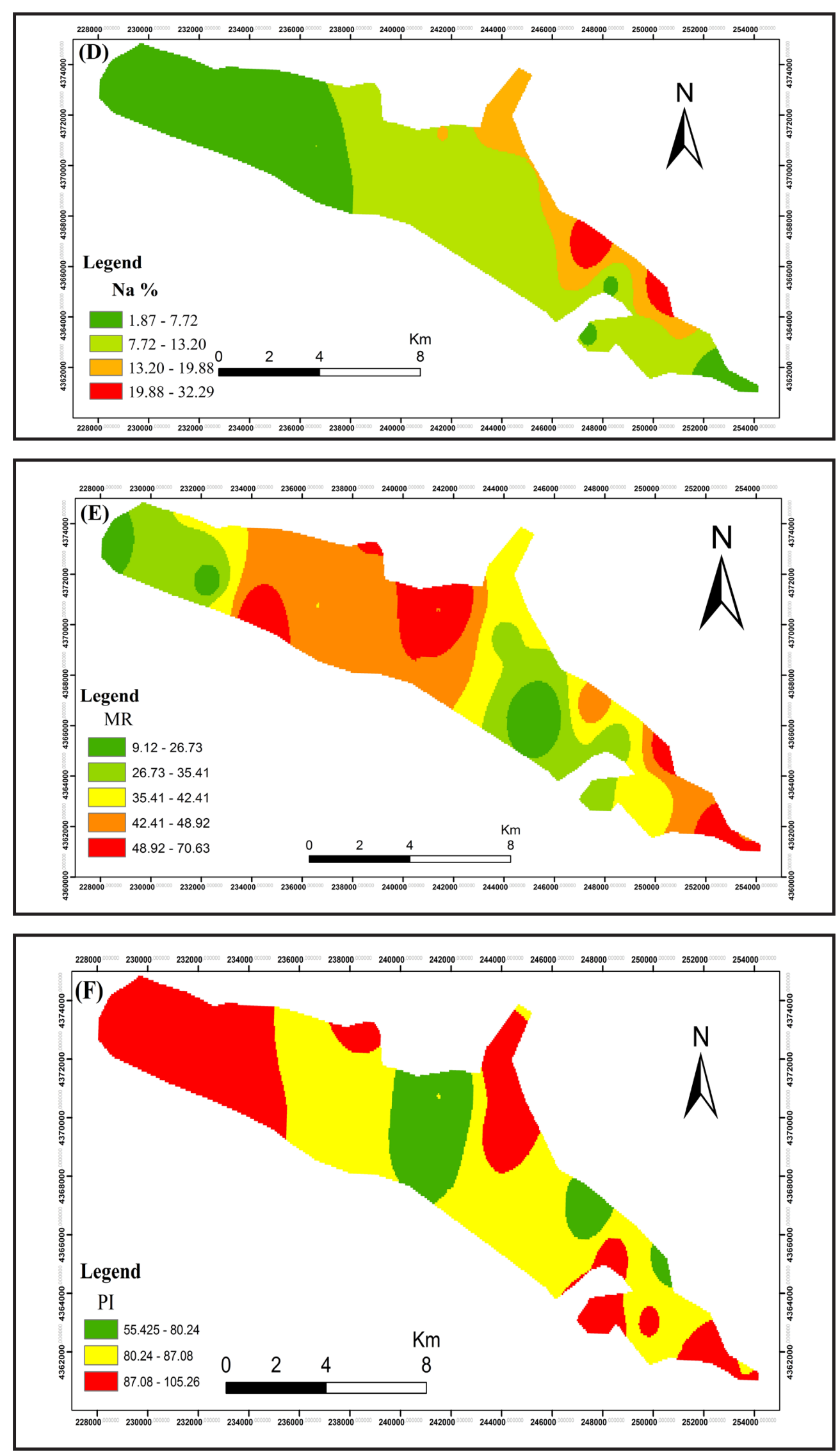

Figure 7- Spatial distribution of average values of two sampling seasons: (D) \%Na, (E) MR and (F) PI. 
spatial distribution map of average PI values of the two different seasons is given in figure $7 \mathrm{~F}$ below and Northwest part of the plain is the safest.

\subsubsection{Kelley Index (KI)}

Kelley Index is expressed as the level of sodium ion measured against calsium and magnesium ions, and it is used to rate irrigation waters (Kelley, 1940;
Paliwal, 1967). All the tested samples of the present study area classified as good because $100 \%$ of the KI values fall within the permissible limit of 1 , indicating the good quality of the groundwater for irrigation purpose (Tables 6 and 7). The Spatial distribution map of average KI value for two sampling seasons is given by figure $8 \mathrm{G}$ below and the safest part of the area is shown in Northwest.
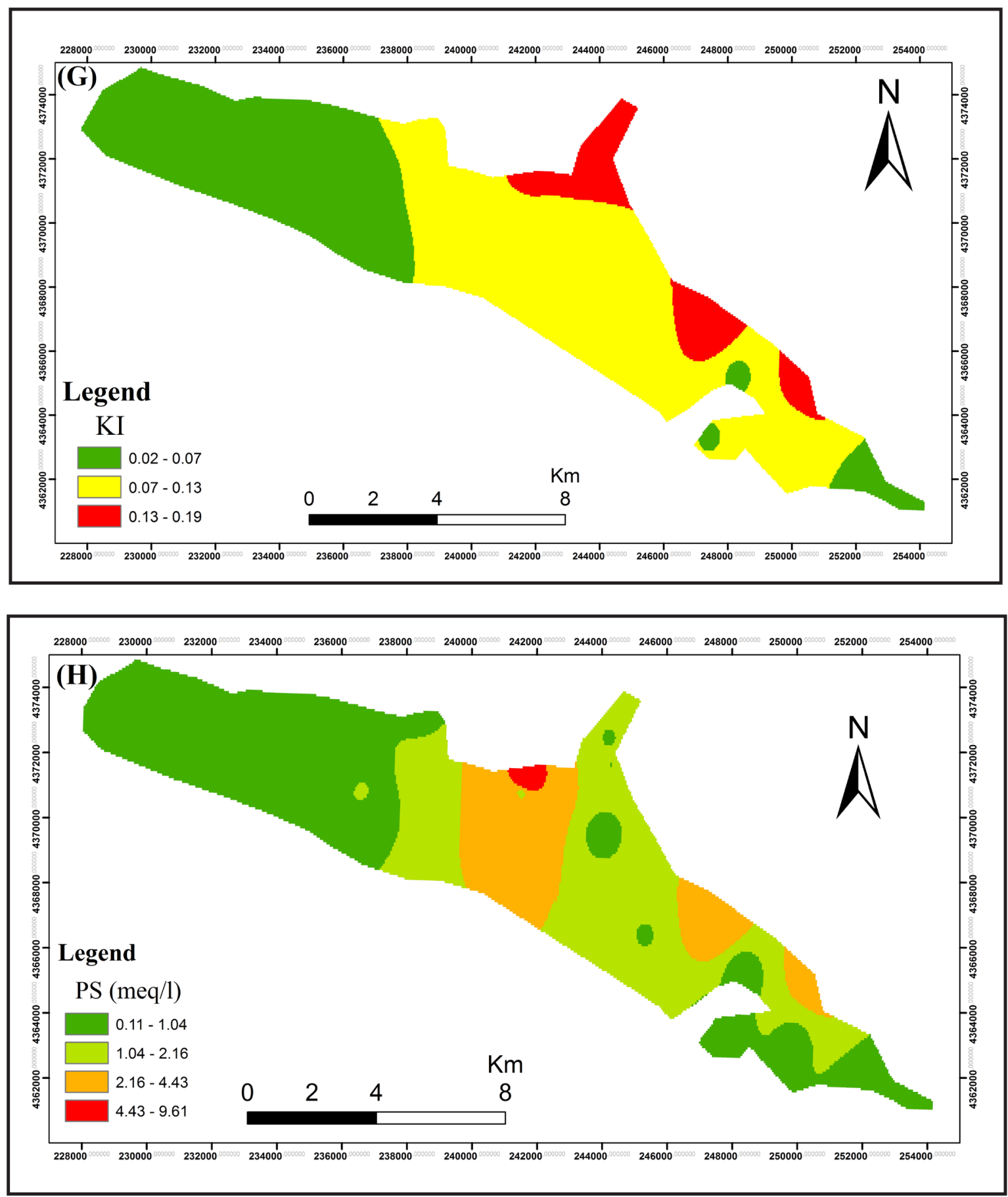

Figure 8- Spatial distribution of average values of two sampling seasons: (G) KI, (H) PS (meq/l) and (I) TH (mg/l). 


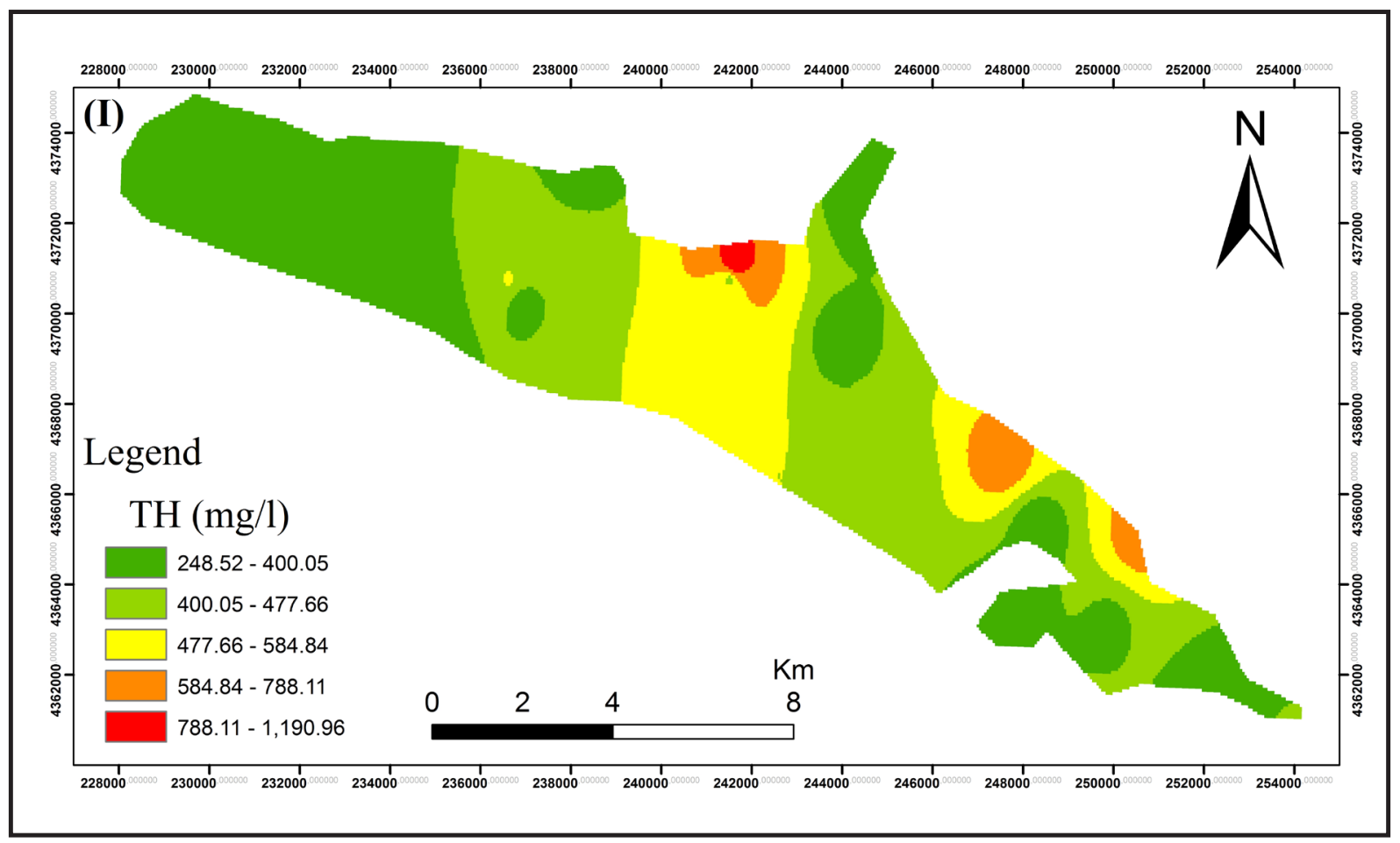

Figure 8- (continued)

\subsubsection{Potential Salinity (PS)}

Potential salinity is another parameter used for the classification of water for irrigation purpose. Potential salinity value less than $3 \mathrm{meq} / \mathrm{l}$ is suitable for irrigation purpose. Results from study area ranged from 0.11 to $11.67 \mathrm{meq} / \mathrm{L}$ (Table 6 ). The results of PS suggest that $85.2 \%$ of waters of the study area are suitable for irrigation. The average potential salinity spatial distribution map of two sampling seasons is produced for the area (Figure $8 \mathrm{H}$ ).

\subsubsection{Total Hardness $\left(\mathrm{TH}_{\mathrm{mg} / \mathrm{l}}\right)$}

Hard water is caused by high levels of calcium and magnesium carbonates. It is expressed as the total concentration of $\mathrm{Ca}^{2+}$ and $\mathrm{Mg}^{2+}$ as milligrams per liter equivalent $\mathrm{CaCO}_{3}$ (Todd, 1980) The values of total hardness ranged from 247.63 to 1363.09 $\mathrm{mg} / \mathrm{l}$ measured for the two different seasons (Table 6). It was witnessed that there was direct correlation $\mathrm{TH}$ with $\mathrm{Ca}^{2+}$ and $\mathrm{Mg}^{2+}$ values as the surface and groundwater in this area was very hard in nature indicating the presence of $\mathrm{HCO}_{3}^{-}, \mathrm{Ca}^{2+}$ and $\mathrm{Mg}^{2+}$ ions concentrations. The plain's spatial distribution map of average TH (mg/l) value for two sampling seasons is given in figure $8 \mathrm{I}$ here under and the total hardness of the water increases towards northwest of the plain.

\section{Discussion}

Most of Kütahya plain groundwaters are $\mathrm{Ca}$ $\mathrm{Mg} / \mathrm{Mg}-\mathrm{Ca}-\mathrm{HCO}_{3}$ water types which shows that geology appears to have greater influence on the chemical transformation of the groundwater resources, compared to any possible effects due to the anthropogenic activities within the study area.

The high value hardness in the waters is resulted from the alluvial and limestone aquifer of Emet formation, which is the main aquifer in the study area.

The $\mathrm{Mg}^{2+}$ ratio values $29.6 \%$ of the water samples are unsuitable for irrigation. This can be explained by excess amount of $\mathrm{Mg}^{2+}$ in the samples. The observed high $\mathrm{Mg}^{2+}$ ion concentration resuled from the dolomite rocks and alluvial aquifer alkalinization of groundwater by leaching from organic fertilisers in weathered soils.

According to the calculated parameters (SAR, RSC, \%Na, PI, KI, MR, PS ve TH) surface and groundwaters of northwest of the study area are less affected by urban wastewater, industrial wastewater and agricultural activities than other area and is good for irrigation purpose than waters from the rest part of the study area. In addition, northwest of the study area is constantly fed by Enne Dam which results in decreasing of ion concentration of surface and shallow groundwater. 
The water chemistry of Kütahya plain is very important and needs care because the Porsuk Dam which uses as drinking water for both Kütahya and Eskişehir cities is situated in downstream. So any activities such as: agricultural activities, use of fertilizers, agricultural spraying, factory wastes and waste storage done in Kütahya plain should be controled.

\section{Acknowledgements}

This paper constitutes part of an on-going $\mathrm{PhD}$ research of the corresponding author, sponsored by the Ankara University Reaserch Institute. The author would like to express his thank and gratitude to the Reaserch Institute Office.

Received: 31.10.2014 Accepted: 11.02.2015

Published: June 2015

\section{References}

Aghazadeh, N., Mogaddam, A.A. 2010. Assessment of groundwater quality and its suitability for drinking and agricultural uses in the Oshnavieharea, Northwest of Iran. Journal of Environmental Protection 1, 30-40.

APHA, 1989. Standard methods for the examination of water and wastewater. $17^{\text {th }}$ edition, American Public Health Association, Washington D.C., 1,268 p.

Appelo, C.A.J., Postma, D. 1994. Geochemistry. Groundwater and Pollution. Rotterdam, A.A. Balkema, $521 \mathrm{p}$.

Ayers, R.S., Westcot, D.W. 1985. Water quality for agriculture. Irrigation and drainage paper No. 29. Food and agriculture organization of the United Nations. Rome, 1-117.

Berhe, B.A., Çelik, M., Dokuz, U.E. 2014. Determination of aquifer properties of the Kütahya plain from a pumping and recovery test data. $67^{\text {th }}$ Geological Congress of Turkey, 14-18 April 2014, Ankara, 324-325.

Çelik, M., Ünsal, N., Tüfenkçi, O.O., Bolat, S. 2008. Assessment of water quality of the Lake Seyfe basin, Kırşehir, Turkey, Environmental Geology, 55, 559-569.

Doneen, L.D. 1964. Notes on water quality in agriculture. Published as a water science and engineering paper 4001, Department of Water Science and Engineering, University of California.

Doneen, L.D. 1966. Water quality requirement for agriculture. Proc. National Sym. Quality Standards for Natural Waters. University of Michigan, Ann. Report, 213- 218.
DSİ, 1981. Kütahya ve Köprüören Ovaları Hidrojeolojik Etüt Raporu, 65, say, Devlet Su Işsleri Genel Müdürlüğü, 64 s, Ankara.

DSİ, 2003. Kütahya Ovası Karst Hidrojeolojisi Ara Raporu, Devlet Su Işleri, $41 \mathrm{~s}$, Eskişehir.

Dufor C.N., Becker, E., 1964. Public water supplies of the 100 largestcities in the United States, 1962: U.S. Geological Survey, Water-Supply Paper 1812.

Hem, J.D. 1991. Study and interpretation of the chemical characteristics of natural waters. Book 2254, third edition. Scientific Publishers, Jodhpur.

Johnson, G., Zhang, H. 1990. Classification of Irrigation Water Quality, Oklahoma cooperative extension fact sheets (available at http://www.osuextra.com).

Joshi, D.M., Kumar, A., Agrawal. N. 2009. Assessment of the irrigation water quality of river Ganga in Haridwar District India. Journal of Chemistry 2(2), 285-292.

Kelley, W.P. 1940. Permissible composition and concentration of irrigation waters, Proc. ASCE 66.

Kelley, W.P. 1963. Use of saline irrigation water. Soil science., 95(6), 385-391.

Naoum, S., Tsanis, I.K. 2004. Ranking of spatial interpolation techniques using a GIS-based DSS. Global Nest: the International journal 6(1), 1-20.

Özburan, M. 2009. Neotectonic investigation of Kütahya and its surrounding. Ph.D. thesis, Kocaeli University, 227 p. Izmit (Unpublished).

Paliwal, K.V., 1967. Effect of gypsum application on the quality of irrigation waters. The Madras agricultural journal 59, 646-647.

Paliwal, K.V. 1972. Irrigation with saline water. New Delhi, IARI, 198p.

Piper, A.M. 1944. A graphical procedure in the geochemical interpretation of water analysis. Transactions, American Geophysical Union 25, 914-928.

Ragunath, H.M., 1987. Groundwater, second ed. Wiley Eastern Ltd, New Delhi, 456p.

Raju N.J., 2007. Hydrogeochemical parameters for assessment of groundwater quality in the upper Gunjanaeru River basin, Cuddapah District, Andhra Predesh, South India, Environmental Geology, 52, 1067-1074.

Richards, L.A., 1954. Diagnosis and Improvement of saline and alkali soils. Agric. Handbook 60, USDA \& IBH Publishing Company Limited, New Delhi, India. 98- 99.

Rowe, D.R., Abdel-Magid, I.M., 1995. Handbook of Wastewater Reclamation and reuse. CRC Press, Inc. $550 \mathrm{p}$.

Schoeller H., 1967. Geochemistry of groundwater. An international guide for research and practice, UNESCO, 15, 1-18. 
Tank, D.K., Chandel, C.P.S., 2010. Analysis of the major ion constituents in groundwater of Jaipur City. Nature and Science, 8(10), 1-7.

Todd, D.K., 1960. Groundwater Hydrology. $1^{\text {st }}$ ed., John Wiley and Sons, Inc, 336p.

Todd, D.K., 1980. Groundwater Hydrology. $2^{\text {nd }}$ ed., John Wiley and Sons, New York, 535p.
WHO, 1993. Guidelines for Drinking-Water Quality - $2^{\text {nd }}$ Edition -Volume 1 - Recommendations, Geneva.

Wilcox, L.V. 1950. Classification and Use of Irrigation Waters. Department of Agriculture, United States, Circular No. 696, 16 p, Washington D.C.

Wilcox, L.V. 1955. Classification and use of Irrigation Waters. U.S. Dept. of Agric., Circular No. 696, 19 p, Washington D.C. 